\title{
Linear magnitudes estimated via expense of incompletely defined potential energy were likely overestimated by over $3.48 \%$
}

\author{
Jakub Czajko \\ Science/Mathematics Education Department \\ Southern University and A\&M College Baton Rouge, LA, USA \\ E-mail address: sunswing77@netscape.net
}

\begin{abstract}
Since former definition of work done by any radial/center-bound (central) force field (and consequently thus also of the corresponding to it expense of potential energy of the field) was incompletely defined (so that these two basic notions were valid only for purely radial phenomena), some indirect estimations of those linear magnitudes that relied on the former (incomplete yet always presumed as total) potential energy may have been overestimated. New, operationally complete and thus mathematically lawful definition of total rate of work done by the field implies presence of a certain (experimentally observed but formerly quite unanticipated and thus routinely unaccounted for) nonradial angular contribution to the total potential energy. Hence some previous calculations of those linear magnitudes, which were indirectly estimated via expense of potential energy spent on the work done, may have been quite inadvertently overrated by over $3.48 \%$. This was because the extra potential energy that is spent on twisting the path that is deflected by the source of the field was disregarded in the former, incomplete definition of work done, even though such nonradial twisting is generally required by proven Frenet-Serret formulas of differential geometry. This present assessment is based upon purely mathematical premises, but similar prior nonradial angular formula utilized here has already retrodicted the $10.56 \%$ excess over Einstein's prediction of deflection of light that was observed in several unbiased experiments, and it has reconciled some other experiments that could neither be explained nor reconciled by general theory of relativity, which, as radial by design, does not account for nonradial or mixed phenomena.
\end{abstract}

Keywords: Potential energy; work done; linear magnitudes; nonradial angular effects

\section{INTRODUCTION}

Since rate of work done and the corresponding to it potential energy of central (i.e. radial/center-bound) force fields used to be mathematically misdefined [1,2], which caused over $25 \%$ latitude-dependent discrepancies in some solar spectra and also over $10.56 \%$ excess over Einstein's prediction of deflection of light (which is essentially phenomenon of mixed radial and nonradial angular nature) [3], the faulty work done can also cause distortion of some estimates of those linear magnitudes that were indirectly estimated via the field's potential energy spent on the work done.

Pretty common justification for neglecting all other than purely radial effects of the usual central radial/center-bound stationary force fields (such as gravitational or electrostatic) often invoked the traditional dictum that only the component of force parallel to displacement 
can do work [4], which was quite incorrectly interpreted as implying that the radial force fields can exhibit no other than the usual radial effects.

Although center-bound force field vectors act only in radial directions, when the local field has more than just one source (such as engines of a spacecraft, or perhaps laser pulses directed at a particle, or extra force fields due to presence of nearby stars - such as our Sun which deflect paths of particles ejected from distant stars) beside the primary (or original) mass source of the field, the effective force vectors can have also some nonradial linear or nonradial angular (or both) subcomponents (with respect to gravity center of the locally dominant field) that are not pointing towards the local (here Sun's) center of gravity. Henceforth by 'field' (if unqualified) I shall always mean the - assumed here as practically stationary for the sake of simplicity - (originally having just one single global center) radial/center-bound local force field, regardless of whether it is of gravitational, electromagnetic or electrostatic nature.

Even though gravitational field of the distant star that ejected the photon (which is then deflected by our Sun) may be stronger than that of the Sun, the Sun's field is locally dominant because near our Sun the distant star's field is vanishingly small. But the impact of the distant star's field had been imparted on and is carried by the ejected photon. The impact is visible in the spectra whose main component is the radial gravitational redshift that depends on difference in the gravitational potentials at the star's surface and at the location of the observer/interceptor [5]. The spectra can have also some other than radial contributions $[3,6]$.

Although the dictum is true for a standalone force, the conclusion is not really adequate for radial force fields, because one has to evaluate interactions of all the directional subcomponents of the other neighboring force vectors rather than only the resultant vector tied to the test particle that moves through (and thus is affected by) the field. For it is not really the resultant/combined force field vector that actually acts on the particle, but each of its orthogonal coordinate subcomponents (when cast in the reference frame of the moving particle) acts (and varies) quite independently of all other coordinate subcomponents of the effective resultant vector [7].

For when a test mass/particle moves through the field, the interacting potentials of the field and that of the mass keep on continually rearranging their positions (and thus also their magnitudes with their positions) as well as their angles with respect to each other. The momentary impacts of all their interactions along the trajectory path would have to be dynamically summed (or integrated, in general) over the trajectory path. It is not just matter of a single, one-time operation of simple addition, in which case the resultant effect would be the same as simple sum of its components.

\section{GEOMETRY REVEALS UNSUSPECTED PHYSICAL RELATIONSHIPS}

In his abstract analysis of an arbitrary three-dimensional (3D) curvilinear motion of a trihedron tied to a moving abstract pointlike particle, Frenet has shown that its motion proceeds in a screwlike manner [8,9]. However, this proven abstract fact was just routinely ignored in former (i.e. created before 2000 AD) physics, because rate of work done and thus also its counterpart (i.e. the field's potential energy that must be spent on the work being done by the field on the twisting) had already been fully but incorrectly defined [1].

The path of a ray deflected by the main source of locally dominant gravitational field is also being twisted nonradially whenever it is being curved by the resultant local force field vector, just as if it was moving on a tilted highway [10]. According to Frenet-Serret (F-S) 
formulas of differential geometry an angular nonradial twist is generally unavoidable and thus its presence should not be ignored [11]. This extra nonradial angular twist is distinct from both: the intrinsic spin of a particle and from its orbital angular momentum (even though - as yet another angular effect - it can certainly affect both of the latter two) in that the twist is induced solely by the source mass/charge of the locally dominant field in which the test mass/particle (or perhaps a satellite) moves along nonradial or mixed (i.e. partly radial and nonradial) path.

The observable fact that angular nonradial twist can arise was not questioned as a physical phenomenon, for it is well known that frequency shift emerges when light beams are rotated [12]. The direct causal connection between rotation of light beams (which means that an angular twist is imposed by the rotation) and their frequency shift was accepted as undisputable fact confirmed in experiments. It was presumably mathematical difficulty that the former physics, which refused to recognize also the other necessary consequence of the connection, namely that if a force field's source is doing the twisting then an extra portion of the field's potential energy must be spent on the work done by the field on implementing the twist, if overall energy is to be conserved. Differential geometry reveals unsuspected structural relationships.

In reality the compensation for the work done by the field on twisting the particle does not just end there. The source mass of the locally dominant field (such as our Sun deflecting a photon passing in the Sun's vicinity) drains energy from the photon as a payment for both the deflection and the twisting [3]. The interaction between the Sun and photon via their fields is thus fully balanced: the photon pays for its passage from its own pocket (or energy packet). Since the work done by the Sun's field must be paid immediately, the Sun field's potential energy compensates for the work. It is then reimbursed by the passing by it photon whose frequency decreases as it gives a part of its energy to the Sun as a payment for all the work done by the Sun's field. This is the abstract structure underlying energy exchange in fieldbased interactions.

In cases where presence of abstract structures (or viability of the corresponding to them procedures) is uncertain, pure mathematics used to trust intuition instead of relying on clues supplied by curious experiments. Dyson warned that mathematical intuition is dangerous, because many [conceptual] situations in science demand for their understanding not the evasion of thought but thought [13]. Evasive solutions provided by pure mathematics in the past did not resolve issues emerging in the real world, but merely buried them so deep, as to make them almost imperceptible.

An example of circumventing actual issues of physical reality was given by G. Choquet who pointed out that the [well-intended but unsubstantiated] Euclid-Hilbert axiomatization of geometry (which was based on the notions of length, angle and triangle) so marvelously concealed the underlying vector space that the concept of vector remained unrecognized for ages [14]. Although most mathematical axioms were extracted from experience, they oftentimes have been abstracted without any deference to experimental hints suggesting existence of conceptually quite different kind of mathematical (and consequently thus also physical) reality than the abstract one envisioned by pure mathematics, which was seemingly designed with the main goal in mind to ease proofs rather than to predict or retrodict natural phenomena.

According to Poincaré certain laws of concordance can be revealed to us only by experience and even though geometry is not really experimental science, some of our innate/intuitive geometrical ideas are guided by experience, which also gives us the occasion to reflect on them [15]. Presence of angular nonradial effects was visible in some experiments $[3,10.12]$ and is implied by geometrical theories $[1,2,11]$. 


\section{FORMERLY MISDEFINED WORK DONE AND POTENTIAL ENERGY}

Nevertheless, the angular twist was never really considered in former physics, because previous definition of work done rate was operationally incomplete and thus precluded any other than just purely radial effects. The angular twist requires some extra potential energy to be spent on the work done by the field on implementing the twisting. Since expense of potential energy used to be equated only with radial rate of the work done, because it was misdefined as $\mathrm{dU}=\boldsymbol{F} \bullet d \boldsymbol{r}$, the nonradial angular twist was routinely disregarded (and thus its effects observed in some experiments were customarily ignored) in the former physics $[1,2,10,11]$. For according to proven (and thus absolutely mandatory) product differentiation rule (PDR) the rate of work done should be defined as $\mathrm{dW}(\boldsymbol{F} \bullet \boldsymbol{r}):=\mathrm{d}(\boldsymbol{F} \bullet \boldsymbol{r})=\boldsymbol{F} \bullet d \boldsymbol{r}+\boldsymbol{r} \bullet$ $d \boldsymbol{F}$ instead [1], in order for it to be both conceptually complete as well as operationally correct.

I shall emphasize the fact that although definitions may be tailored to the areas of interest (and expertise) of their authors, they should not be permitted to violate any verified laws of nature or proven rules/laws of mathematics, which are prototypes of certain (even if unknown yet) laws of nature. Hence no theory should be allowed to waive any rigorously proven laws or rules, regardless of whether they are enforced by some unambiguous theories of physics or were established by abstract deductions and/or derivations accomplished by mathematics. Definitions should not permit thus performing any illegitimate mathematical operations. There is also no excuse for legitimizing unlawful definitions by devising phony theorems [1].

\section{SYNTHTIC MATHEMATICS LAYS FOUNDATIONS FOR PHYSICS}

Although even brilliant scientists, such as Gell-Mann, for one, refused to call it science [16], applied mathematics (as opposed to the pure mathematics that is often viewed as a sort of art) is actually the only science really worthy of this designation, whereas all the other exact sciences provide just comments, which could amount to empty talk without support from experiment-driven (new) synthetic mathematics. The fact is, however, that without such comments supplied by theories of exact and semi-exact sciences mathematics could be conceptually lost. The dependence is thus mutual, but its structural foundations and the corresponding to them procedures of mathematical framework of physics should be laid by mathematics.

Since physical reality cannot be ever deduced from any a priori philosophical conceptions [17], perhaps it really is a giant abstract mathematical structure [18]. For it should not originate in the physics itself, which is supposed to verify viability of the structure and therefore it should not lay its own abstract structural foundations. From this point of view, most basic foundations of the physical reality we live in should be external to physics. Otherwise a chain of theoretical vicious circles could be created when physics would both invent and then also validate its own creations. To avoid this clear conflict of interests, physics should not unduly influence its own foundations by trying to make them appear "normal" by contemporary standards of normalcy and/or in line with common sense expectations.

At the present (essentially classical) depth of inquiry into gravitational (and other force field-related) phenomena I assume that field forces materialize as the result of clashing potentials of mass/charge sources of their respective local fields $[10,19]$. 


\section{CORRECT DEFINITION OF WORK DONE BY FIELDS}

The former (radial-only) definition of rate of work done disregarded the product differentiation rule which has been proven [20] and therefore is mandatory [1,2]. If the rate of work done function $\mathrm{dW}()$ of the work done $\mathrm{W}$ by a force field vector $\boldsymbol{F}$ is defined in an operationally complete and thus mathematically legitimate way [1] as

$$
\mathrm{dW}(\boldsymbol{F} \bullet \boldsymbol{r}):=\mathrm{d}(\boldsymbol{F} \bullet \boldsymbol{r})=\boldsymbol{F} \bullet d \boldsymbol{r}+\boldsymbol{r} \bullet \mathrm{d} \boldsymbol{F}
$$

then operationally complete total rate of the work done by the force field is given by

$$
\mathrm{dW}=2 \mathrm{Frsin} 2 \alpha \mathrm{d} \alpha-\cos 2 \alpha(\mathrm{Fdr}+\mathrm{rdF})
$$

which contains the nonradial angular twist $2 \mathrm{Frsin} 2 \alpha \mathrm{d} \alpha$ that is acquired only along equipotential surfaces [3] whereon the magnitude $r=|\boldsymbol{r}|$ of the pointing radius and the magnitude of attractive Newtonian gravitational force $F=|\boldsymbol{F}|=-\frac{G M m}{r^{2}}$ remain constant, and so is the gravitational constant $\mathrm{G}$ and also the interacting masses $\mathrm{M}$ and $\mathrm{m}$ for the purpose of this paper.

Notice that when properly considered at the point of impact (i.e. where the small test mass $\mathrm{m}$ is located within the field that is due to the bigger mass $\mathrm{M}$ ) the angular nonradial subcomponent emerges quite naturally (i.e. by itself, without ever being postulated) [1,2], in accordance with to the F-S formulas [11]. Strict adherence to proven mathematical rules/laws ensures the viability of conceptual representation of the notion of work done and offers its operationally sound mathematical expression.

Even though it is determined by distance traveled along an equipotential surface, this nonradial angular twist is actually quasi-static effect, which is independent of the parameters of the motion that is required for the prerequisite transport of the particle or satellite within the field. The pointing vector $\boldsymbol{r}$ links the gravity center of the local field with generic point on the trajectory path of the particle that moves in the field and $\alpha$ is the (originally planar) spherical angle between the pointing vector and the line segment that links the perihelion with the gravity center of the local field [1]. In the sense $\alpha$ is the angle of visibility of the path.

The mathematically legitimate and thus operationally correct eq. (2) contains two clearly nonradial terms (angular and linear) in addition to the usual radial linear term $\boldsymbol{F} \bullet \mathrm{d} \boldsymbol{r}$. These new extra terms are definitely nonradial, for the radial distance $r=|r|$ is fixed therein and therefore the angular rate $\mathrm{d} \alpha$ must be varying nonradially, which can only happen along local equipotential surfaces whereon the distance $r$ remains constant with respect to the local gravity center.

In addition to being illegitimately defined, the former, operationally incomplete rate of work done (and so also the corresponding to it potential energy) could never yield total potential energy spent on the work done by the force field, regardless of whether it is gravitational, electromagnetic or electrostatic. Since we always tend to assume that Hamiltonian function comprises total potential energy in order for the energy to remain balanced and thus conserved in interactions, which is especially important in quantum mechanics $(\mathrm{QM})$ - the former, incompletely defined potential energy that compensates the work done by the local field caused some (very rarely acknowledged in physics but recognizable in applied mathematics) deficiencies. The best example of that could be inefficiency of Schrödinger equation (which is often dubbed as the "workhorse" of the QM) 
when applied to atoms whose nuclei contain more than just one proton, because this means presence of a definitely multisourced field which may exhibit thus certain nonradial effects in accordance with the F-S formulas. Formerly, the workhorse used to be fed with incomplete potential (energy).

When yet another mass source affects (or contributes to) the local force field, the formerly ignored angular energy term cannot be neglected anymore, for equipotential effects of the field's interactions always depend on angles too. Several experiments have already confirmed existence of clearly angular nonradial effects acquired along equipotential surfaces of the locally dominant gravitational force field $[3,10]$.

From the eq. (2) a new nonradial angular function $\mathrm{W}_{\mathrm{rQ}}()$ for rate of work done has already been derived for the local equipotential surface $\alpha$ that is codetermined by the pointing radius $r$ and the perihelion radius $r_{p}$ of trajectory path of the moving mass $m$

$\mathrm{W}_{\mathrm{rQ}}(\mathrm{Q}(\lambda))=\frac{-4 \mathrm{kGMmr} \mathrm{p}_{\mathrm{p}}^{2} \lambda}{3 \mathrm{Qr}}$

where $\mathrm{Q}$ is density of matter of the source mass $\mathrm{M}$ that creates the locally dominant force field, $m$ is negligibly small mass of the test body passing by the mass $M$ near its surface and $\lambda$ $=\mathrm{r} \theta$ is the angular path (on an equipotential surface surrounding the gravity center of the local gravitational field that is due to the mass $\mathrm{M}$ ). The angular path $\lambda$ corresponds to curvilinear, in general, spherical angle $\theta$ that determines the path $\lambda$ on the surface [2]. Note that $\alpha$ is the (originally planar spherical, but then - after it is twisted - it becomes also spheroidal spherical) angle of visibility of the trajectory path spreading between the pointing radius $r$ and the radius of perihelion $r_{p}$. Notice that only the equipotential distance $\lambda=r \theta$ and the corresponding to it angle $\theta$ pertain directly to the field-induced angular nonradial twisting. The pointing radius $\mathbf{r}$ points to generic moving point on the trajectory path.

The right-hand side (RHS) term of the eq. (3) is more comprehensive/detailed than the previously derived nonradial angular formula, which did not comprise any reference to the radius of perihelion; but the eq. (3) is in conceptual sync with the previous angular nonradial formula [3].

The angular nonradial eq. (3) has been derived from mathematical premises. It supersedes thus my previously obtained formula, which was less detailed because it has been deduced from experimental results [3] by taking clues primarily from the experiments [21] and [22]. The eq. (3) vindicates thus codependence of the angular nonradial effects of the radial gravity field on exposure (of the test mass $\mathrm{m}$ ) to the constant density of matter Q (of the field's source mass M), which has already been introduced in the previously derived formula [3].

Since the previous formula has already been confirmed by several independent experiments, and because it has already successfully retrodicted over $10.56 \%$ of the experimentally observed excess [23] over Einstein's prediction of deflection of light, I shall rather use this (experimentally verified and thus fairly viable) number for the estimate to be presented in what follows. Although the deflection of light is partly an angular nonradial effect by its very nature, Einstein refused to consider any possible tangential effects of the usual radial gravity field [24], whose presence would imply dependence of the deflection on a spherical angle (and thus on the corresponding to it spherical distance) that is measured along equipotential surfaces.

The determination of angular nonradial effects also by the perihelion radius of the trajectory path of a satellite (represented here by the test mass $\mathrm{m}$ ) is very subtle mathematical 
feature, which only slightly modifies the primary dependence on the radial distance. It is inconsequential for general, rough estimates.

Although the new comprehensive angular nonradial formula (3) is conceptually similar to the previous experiment-driven formula [3], it has not been experimentally verified yet, and therefore - for the estimations calculated at this time - I shall rather use the previously derived factor of $10.56 \%$ instead, because it has already included exposure to the density of matter $\mathrm{Q}(\lambda)$ and has been experimentally confirmed - [3,10,23]. The most important point is the dependence on the density of matter.

\section{LINEAR MAGNITUDES ESTIMATED INDIRECTLY VIA FORMER MISDEFINED POTENTIAL ENERGY WERE LIKELY OVERRATED}

Presence of formerly disregarded angular nonradial contributions to work done by a radial/center-bound force field, and to the corresponding to it potential energy, suggests that some previous estimates of some indirectly calculated/estimated linear magnitudes, such as cosmological distance based on redshifts found in the spectra of light rays emitted by a distant star, which are indirectly tied to potential energy of the local (deflecting them) field, or perhaps radius of the proton in atomic nucleus whose size was indirectly estimated via expense of its electric field's potential energy spent on the encircling it electron (or muon), may have been inadvertently overrated.

This is because the incomplete former definition of work done that corresponds to the force field's potential energy spent on the work done by the field was routinely assumed as the total potential energy involved in the field-based interactions, even though it was obviously only the radial part of the potential energy involved therein.

Although the eq. (3) and the previously derived, experiment-driven formula [3] pertain to gravitational force fields, all radial force fields (regardless of whether they are gravitational or approximately electrostatic) can generate the quasi-static angular nonradial effects because these effects are mathematically necessary. Emergence of angular nonradial twist is universal mathematical feature [11] and thus is applicable to all radial/center-bound force fields regardless of their character.

The extra frequency decrease found in the experiments conducted by Sadeh with rays deflected by Sun [21] and with radio waves bent by Earth [22] was reconciled when Sadeh's experiment-driven equations have been augmented by respective densities of matter of these two celestial bodies $[3,10]$, the need for which was also found in classical radial potential theory applied to heat flow [25].

These facts indicate that exposure to density of matter (which can measured by specific gravity, if it is constant and uniformly distributed) of the mass that generates the locally dominant gravitational field affects phenomena happening within the field in similar manner as specific heat affects the work being done by flow of heat. Yet former mathematics did not support the ideas hinted at by the two Sadeh experiments as well as by the classical potential theory deployed in thermodynamics.

As a matter of fact, the so-called path independence theorem (PIT) of work done has been invented (though never really proven) to preclude the particular venue of prospective inquiry into nonradial gravitational phenomena [1], or perhaps to simply give a "lame" excuse to those who (like A. Mercier in [26,27]) explicitly voiced their doubts about conceptual soundness of the former, incomplete mathematical representation of potential energy. Yet (to the best of my knowledge) the operational incompleteness of the former definition of work 
done and potential energy was not recognized in former physics until it has eventually been challenged in [1].

Hence if the error of some indirectly estimated linear magnitudes via expense of the potential energy of a field on the work done by the field was due to the (formerly neglected) nonradial angular twist of at least $10.56 \%$, then from the simple relation

$\frac{100-10.56 / \pi}{100}=\frac{100}{\mathrm{x}}$

we obtain the possible overestimate $\mathrm{x}$ and the net defect $\Delta \mathrm{x}$ of the linear magnitude

$$
\mathrm{x} \cong 103.48 \% \Rightarrow \Delta \mathrm{x} \cong 3.48 \%
$$

which sets a lower bound. Hence actual errors could be greater than that, of course.

This overestimate is pretty close to the recently found defect of $\sim 4 \%$ of diameter of the (reported as allegedly shrinking) proton that has been found during indirect measurement with the help of a laser beam, which (by adding some extra energy to the interaction between the proton and the encircling it muon $[28,29]$ should have induced thus also some mandatory (yet routinely unaccounted for in former physics) extra nonradial angular effects.

The use of laser, whose impact is generally equivalent to presence of yet another, hence quite distinct additional source of the effective (if approximately stationary - i.e. practically almost electrostatic) local force field that was initially due to the proton alone. This makes the resultant force vectors noncentral with respect to the initial center of the proton's field, in which case some nonradial components of the vectors must (according to the F-S formulas of differential geometry) necessarily arise and therefore their impact should not be discarded without compelling reason.

Since presence of some nonradial effects other than the angular nonradial twist discussed above is also possible, some linear magnitudes were likely overestimated by at least $3.48 \%$ due just to the former, incomplete operational definition of work done by the radial force field and thus also of the field's potential energy that is spent on (or corresponds to) the work done by the field on the twisting.

\section{CONCLUSIONS}

Since former definition of rate of work done (and thus also the corresponding to it potential energy) was defined in operationally incomplete way, linear magnitudes estimated via expense of that incomplete (yet routinely assumed as total) potential energy spent on the angular nonradial part of work done by the field may have been overrated by at least $3.48 \%$, due to the traditional neglect of the angular nonradial contributions to potential energy (in any radial/center-bound force fields, whether of quasi-static gravitational or electromagnetic or electrostatic character) that are due to the twisting of a passing by satellite's path, which is absolutely necessary according to Frenet-Serret formulas of differential geometry.

The aforeshown estimate pertains to quasi-static gravitational (or approximately electrostatic) field. It did not take into account any dynamic factors. The essentially quasistatic angular nonradial component of the potential energy is the part that was formerly unrecognized and therefore routinely ignored. 
Center-bound force fields having (beside the main source of the locally dominant force field) also some additional distinct sources that also contribute to the effective resultant local force field (such as engines of a spacecraft, or perhaps laser pulses, or the energy imparted by the distant star from which a particle has been emitted) which cannot be quite meaningfully amalgamated into as if common (i.e. due to single local source) radial/center-bound force field, should include the impact of both the radial and all nonradial (angular and/or linear) potentials from each of these sources.

Hence the formerly ignored nonradial contributions to total energy of interactions within radial/center-bound force fields are likely to give, if included, more accurate but presumably also somewhat smaller estimates for those linear magnitudes whose indirect measurement was based upon the former, incompletely defined work done and potential energy.

\section{References}

[1] Czajko J., Stud. Math. Sci. 7(2) (2013) 25-39.

[2] Czajko J., Stud. Math. Sci. 7(2) (2013) 40-54.

[3] Czajko J., Chaos Solit. Fract. 11 (2000) 2001-2016.

[4] Lord Kelvin, Guthrie P., Treatise on natural philosophy. Cambridge: At the Univ. Press, 1903, p. 238f.

[5] Beiser A., Concepts of modern physics. New York: McGraw-Hill, 1973, p. $67 \mathrm{ff}$.

[6] Czajko J., Chaos, Solit. Fract. 11 (2000) 1983-1992.

[7] Sokolnikoff I. S., Sokolnikoff E. S., Higher mathematics for engineers and physicists. New York: McGraw-Hill, 1941, p. 218.

[8] Struik D. J., Lectures on classical differential geometry. Dover, New York 1988, p. 18f.

[9] Sauer R., Differenzgeometrie. Berlin: Springer, 1970, p. 160.

[10] Czajko J., International Letters of Chemistry, Physics and Astronomy 11(2) (2014) 89-105.

[11] Czajko J., Appl. Phys. Res. 3(1) (2011) 2-7.

[12] Padgett M. J., Allen L., Opt. Quantum Electron. 31 (1999) 1-12.

[13] Newton R. G., Thinking about physics. Princeton, NJ: Princeton Univ. Press, 2000, p.77.

[14] Choquet G., Geometry in modern setting. Paris: Hermann, 1969, p. 14.

[15] Poincaré H., On the foundations of geometry. [pp.117-146 in Pesic P. (Ed.) Beyond geometry. Classic papers from Riemann to Einstein. Mineola, NY: Dover, 2007, see pp. 135, 144].

[16] Gell-Mann M., The quark and the jaguar. New York: W.H. Freeman and Co., 1994, p. 108.

[17] Bohr N., Phys. Rev. 48 (1935) 696-702.

[18] Tegmark M. Our mathematical universe. New York: Alfred A. Knopf, 2014, pp. 260, 280. 
[19] Czajko J., Chaos Solit. Fract. 19 (2004) 479-502.

[20] Bers L., Calculus I. New York: Holt, 1967, p. $216 \mathrm{f}$.

[21] Sadeh D., Knowles S. H., Yaplee B. S., Science 159 (1968) 307-308.

[22] Sadeh D., Knowles S., Au B., Science 161 (1968) 567-569.

[23] Merat P., Astron. Astrophys. 32 (1974) 471-475.

[24] Einstein A., The Foundations of the General Theory of Relativity. [pp.111-164 in: H.A. Lorentz et al. The principle of relativity. Dover, New York 1923, see p.161].

[25] Kellog O. D., Foundations of potential theory. Berlin: Springer, 1929, p. 77f.

[26] Mercier A., Analytical and canonical formalism in physics. North-Holland, Amsterdam 1959, p. 122.

[27] Mercier A., Speculative remarks on physics in general and relativity in particular. [pp.295-303 in: V. De Sabbata, J. Weber (Eds.) Topics in theoretical and experimental gravitation physics. London: Plenum, 1977].

[28] Antognini A., et al. Science 339(6118) (2013) 417-420.

[29] Margolis H. S., Science 339(6118) (2013) 405-406. 